\title{
Measuring Positive and Negative Affect in a School-Based Sample: A Polish Version of the PANAS-C
}

\author{
Monika Wróbel $^{1}$ (D) Maria Finogenow ${ }^{1} \cdot$ Paulina Szymańska $^{1} \cdot$ Jeff Laurent $^{2}$
}

Published online: 1 February 2019

(C) The Author(s) 2019

\begin{abstract}
Students in Grades 4-6 (N=722) completed a Polish-language version of the Positive and Negative Affect Scale for Children (PANAS-C; Laurent et al. Psychological Assessment, 11, 326-338, 1999). The psychometric properties of the Polish PANAS-C were examined using methods employed by other validation studies. Additionally, two alternative models suggested in the literature - a 10-item PANAS-C (Ebesutani et al. Journal of Psychopathology and Behavioral Assessment, 34, 191-203, 2012) and a second-order PANAS-C (Ebesutani et al. Psychological Assessment, 23, 679-691, 2011a) - were tested. Confirmatory factor analyses demonstrated that the 10-item version performed better that the two remaining versions. The analyses for the original 2-factor model and the second-order model suggested that the underlying structure of the PANAS-C, similar to the structure of the original PANAS, may be more complex than initially assumed. The PA and NA scores were related to traditional self-report measures of child anxiety and depression demonstrating that the pattern of correlations was consistent with the tripartite model of affective disorders. Gender and age differences were also consistent with those reported in the literature. Together these results suggest that the Polish-language version adds to the expanding number of translations of the PANAS-C, indicating that the scale can be used with samples from culturally diverse backgrounds.
\end{abstract}

Keywords PANAS-C $\cdot$ Positive affect $\cdot$ Negative affect $\cdot$ Anxiety $\cdot$ Depression

Measurement of affect has long been a central component of emotion research (Diener 1999; Mauss and Robinson 2009). According to one of the most widely held views, popularized by Watson, Tellegen, and colleagues (Watson and Tellegen 1985; Watson and Clark 1984; Zevon and Tellegen 1982), affective space is best represented as two broad, separable factors referred to as Positive Affect (PA) and Negative Affect (NA). While PA reflects pleasant engagement with the environment (e.g., being interested, strong, active), NA is a broad general factor of emotional distress that includes moods like being nervous, miserable, or upset. In an effort to facilitate accurate assessment of these factors, Watson et al. (1988) developed the Positive and Negative Affect Schedule (PANAS) that consists of two 10-item scales (one for PA and one for NA). The PANAS has been shown to be highly

Monika Wróbel

monika.wrobel@uni.lodz.pl

1 Institute of Psychology, University of Lodz, ul. Smugowa 10/12, 91-433 Łódź, Poland

2 Department of Psychology, Western Illinois University, Macomb, IL, USA reliable and valid across diverse samples, time frames (e.g., right now, past month, in general), and languages (e.g., Crawford and Henry 2014; Merz et al. 2013; Rush and Hofer 2014; Terracciano et al. 2003). Moreover, its utility has been demonstrated in research on the tripartite model of anxiety and depression. The model holds that the two disorders share a common component of general emotional distress (negative feelings), but differ in two other components: Absence or substantial reduction of pleasurable experience (lack of positive feelings) is specific to depression, whereas physiological hyperarousal ( $\mathrm{PH})$ is specific to anxiety (Clark and Watson 1991). Thus, anxiety and depression can be differentiated on the basis of the PANAS scores: High levels of NA are common to both disorders and low levels of PA are associated with depression but not with anxiety (Watson and Clark 1995).

It was only a matter of time until a child version of the PANAS, the Positive and Negative Affect Scale for Children (PANAS-C; Laurent et al. 1999), was developed. Its authors aimed to develop PA and NA scales that would be understandable to children. When creating the youth version of the PANAS, Laurent et al. relied on a large pool of PA and NA items taken from the expanded form of the PANAS, the 
PANAS-X (Watson and Clark 1994). The results of the preliminary study conducted among fourth and eighth graders demonstrated that some of these items (including seven items from the original PANAS, e.g., irritable, attentive) might be too difficult for some participants to understand (see also Joiner et al. 1996). Such items were either eliminated or replaced with synonyms, which resulted in an initial, 30-item version of the PANAS-C that was later reduced to 27 items. The time frame adopted in this version was over the past few weeks, which allowed for the measurement of relatively longterm fluctuations in mood (i.e., affective states that keep reoccurring in a child's recent affective experience; Watson 2000). Research demonstrated that the psychometric properties of the PANAS-C were similar to those of the original PANAS. Specifically, exploratory factor analyses yielded two easily interpretable factors - PA and NA - that were highly reliable and valid (Laurent et al. 1999). Moreover, in line with the tripartite model of anxiety and depression, the PANAS-C scores were related to the symptoms of these disorders in a similar way as the original PANAS scores, across both clinical (e.g., Chorpita and Daleiden 2002; Ebesutani et al. 2011a; Hughes and Kendall 2009) and non-clinical samples (e.g., Ebesutani et al. 2011a; Laurent et al. 1999).

At the same time, the PANAS-C has drawn attention from other investigators not directly interested in the tripartite model. For example, the scale has been used in studies of yoga and mood (Felver et al. 2015), psychological mindedness and coping (Roxas and Glenwick 2014), sleep deprivation (Dagys et al. 2012), and physical illness (Sinnamon et al. 2013; Tsao et al. 2014). Additionally, Ebesutani et al. (2012) have recently developed a shorter, 10-item version of the PANAS-C. Although this shorter version taps a less broad variety of affective feelings compared to the full PANAS-C (e.g., the NA scale does not include any adjectives related to guilt), it contains the items recommended for a comprehensive assessment of affect (Watson and Clark 1997). Moreover, the 10-item PANAS-C was found clinically useful for identifying children with anxiety and depression disorders, similar to the longer version (Ebesutani et al. 2012). Hence, although the 10-item PANAS-C cannot be used interchangeably with the original PANAS-C because of its narrowed content, Ebesutani et al. (2012) stress that the 10-item version allows for quick and efficient assessment of affect and thus should be considered when the battery of questionnaires is long and time is limited (e.g., in screening studies).

As psychometric soundness and broad utility of the PANAS-C were being established and the need for crossculturally validated research scales was recognized (Sousa and Rojjanasritat 2011), researchers outside the United States became interested in translating and using the measure. Kiernan et al. (2001) were among the first researchers to translate the PANAS-C into several languages for use with a sample of children from various European countries. The number of children from any particular country was small, so data were combined in analyses. Nonetheless, the PA and NA constructs were supported. Subsequently, others have translated the PANAS-C and used it with specific samples of youth, following the methods adopted by Laurent et al. (1999). Specifically, psychometric properties of the translated PANAS-C scales were tested with exploratory factor analysis to investigate the structure of the scale and hierarchical multiple regression to examine partial correlations of the PA and NA scales with measures of anxiety and depression. For instance, Yamasaki et al. (2006) developed a Japanese version of the PANAS-C. Stevanovic et al. (2013) developed a Serbian translation of the PANAS-C. Italian (Ciucci et al. 2017), Spanish (Casuso et al. 2016), and Chinese (Ting-Ting et al. 2015) translations of the PANAS-C also appear in the literature. Generally, these translated PANAS-C scales eliminate some items based on language variations between the original English version and the particular translated versions; however, the resulting translated PANAS-C scales demonstrated psychometric properties similar to those of the original scale.

The current study adds another foreign language version of the PANAS-C to the child assessment literature by translating the measure into Polish and using the aforementioned methods employed by other validation studies. In line with these studies, we predicted that PA and NA would represent two general, weakly-to-moderately correlated dimensions, and that children in our non-clinical sample would obtain higher scores on the PA scale than on the NA scale. We also examined the relations of the PANAS-C scales to traditional self-report measures of anxiety and depression to provide preliminary evidence of convergent and discriminant validity for the Polish version of the scale. Specifically, we expected that the correlations between the NA scale and these traditional measures would be moderate-to-high and positive. We expected scores on the PA scale to be negatively correlated with scores on self-report measures of anxiety and depression. When controlling for NA, the relationship between PA and depression should remain negative and significant, while the correlation with anxiety should remain small and nonsignificant (Laurent et al. 1999). Finally, based on the observation that affective experiences and other affect-related constructs (e.g., anxiety and depression) differ across age and gender (e.g., Jacques and Mash 2004; Salk et al. 2017; Stevanovic et al. 2013; Yamasaki et al. 2006), we investigated whether the Polish PANAS-C scores, similarly to other language versions, also reflect these differences. Building upon previous research (Ciucci et al. 2017; Jacques and Mash 2004; Stevanovic et al. 2013), we expected that girls would obtain higher scores on the NA scale than boys, and that older students would have higher scores on the NA scale than younger students. With respect to the PA scale, limited evidence suggests that younger girls may obtain higher scores than older girls (Stevanovic et al. 2013). 
Our study also extends previous research in two ways. First, unlike most of the previous studies addressing the properties of the PANAS-C, we examine the structure of the scale more thoroughly by means of confirmatory factor analysis (CFA). Notably, although the PANAS-C has been widely used by researchers since the late 1990s, in-depth analyses of its structure that go beyond exploratory factor analysis are relatively new and paint a somewhat inconsistent picture, supporting either two-dimensional or multidimensional models (e.g., Bushman and Crowley 2010; Ciucci et al. 2017; Ebesutani et al. 2011a, 2011b, 2012). Thus, following the discussion concerning the possible multidimensionality of the NA scale (Gaudreau et al. 2006; Killgore 2000), we investigated whether the NA scale of the PANAS-C could be divided into two strongly correlated components, namely $\mathrm{NA}_{\text {fear }}$ and $\mathrm{NA}_{\text {distress. }}{ }^{1}$ Therefore, in addition to the two-dimensional model proposed by Laurent et al. (1999), we tested an alternative second-order model previously tested only in a U.S. sample (Ebesutani et al. 2011a). Second, following the observation that so far the utility of the 10-item PANAS-C has been demonstrated only in two cultures - the U.S. (Ebesutani et al. 2012) and Spain (Sanmartín et al. 2018) - we examined the properties of this shortened version in our study. Of importance, we adapted this version instead of creating a short form in its strict sense (i.e., by reducing the Polish version of the PANAS-C; Smith et al. 2000), because comparable versions of an already existing scale might facilitate multinational and multicultural research on affect in children (Beaton et al. 2000). Moreover, given that the aforementioned studies conducted in the U.S. and Spain strongly supported the twodimensional structure of the 10-item PANAS-C, we assumed that adding another foreign language version of this scale is important in the light of the ongoing discussion on the underlying structure of the PANAS-C.

\section{Method}

\section{Participants}

The sample consisted of 722 students ( 379 boys, 343 girls) recruited from nine elementary schools randomly selected from a total of 86 elementary schools located in a large city in central Poland. After selecting the schools, we included only the students who were present at school on the day of

\footnotetext{
${ }^{1}$ In addition to the second-order-model, Ebesutani et al. (2011a) tested a bifactor model of NA scale consisting of orthogonal $\mathrm{NA}_{\text {fear }}$ and $\mathrm{NA}_{\text {distress }}$ factors. Although the fit of this model was slightly better than the fit of the second-order model, only 8 out of the $15 \mathrm{NA}$ items loaded on the $\mathrm{NA}_{\text {fear }}$ and $\mathrm{NA}_{\text {distress }}$ factors (the loadings of the 7 remaining items were below .40). Moreover, $\mathrm{NA}_{\text {fear }}$ and $\mathrm{NA}_{\text {distress }}$ were strongly related (Ebesutani et al. 2011a, Figure 2), calling into question the orthogonality of these subdimensions. Thus, we did not test this model in the current study.
}

the study, agreed to participate in it, and whose parents provided written consent (72\% of all parents). The mean age of the students was 11.95 years $(S D=.88)$. Fourth graders made up $30 \%$ of the sample ( $n=217 ; 91$ boys, 126 girls), fifth graders made up $34 \%$ of the sample $(n=245 ; 104$ boys, 141 girls), and sixth graders comprised $36 \%$ of the sample $(n=$ $260 ; 148$ boys, 112 girls).

\section{Measures}

The Positive and Negative Affect Scale for Children (PANAS-C; Laurent et al. 1999) The PANAS-C, initially developed as a 30-item self-report instrument, measures positive affect (PA) and negative affect (NA) in children and adolescents. Laurent et al. reported that the final version of the PANAS-C consists of 27 adjectives describing various affective states (12 PA items, $15 \mathrm{NA}$ items). Students are asked to rate the extent to which they experienced each feeling over the past few weeks, using a 5-point Likert-type scale ranging from very slightly or not at all to extremely. Validation studies using culturally diverse samples (e.g., Ciucci et al. 2017; Kiernan et al. 2001; Stevanovic et al. 2013) have used the initial 30 -item version of the PANAS-C developed by Laurent et al. (1999) and have eliminated some items from the original version. Therefore, we also decided to use the initial 30-item version in the current study.

Three Polish psychologists with a good command of English (an expert in developmental psychology and two experts in psychology of emotions) independently translated the 30 original items of the PANAS-C into Polish. A parallel translation was chosen instead of a typical back translation to ensure that all items would be not only linguistically equivalent to the original ones, but also natural and comprehensible for Polish children (Behr 2017; Gudmundsson 2009). The three versions were carefully compared and discrepancies between them discussed. Thirteen items ( 8 NA and 5 PA) had more than one translation equivalent accepted by all the translators (e.g., blue and lively both had three possible translations). Thus, it was decided to keep them all in the preliminary version of the Polish PANAS-C. This resulted in an initial pool of 45 adjectives.

The pool was then subjected to further analyses, the aim of which was to reduce it back to 30 items by choosing the items that were best understood by children. For this purpose, we carried out a preliminary study similar to the one conducted by Laurent et al. (1999). We presented the list of 45 adjectives to 37 fourth-graders (19 boys and 18 girls) and asked them to rate the understandability of these adjectives on a 3-point scale: $1=I$ don't know what this word means, $2=I$ am not entirely sure what this word means, and $3=I$ know what this word means. For the 13 items with multiple possible translations, the version retained was the one that had the most number of students indicating they knew what the word meant 
$(n \geq 33)$ with no child indicating they did not know the meaning of the word. The selected items formed the initial Polish version of the PANAS-C used in the current research.

\section{State-Trait Anxiety Inventory for Children (STAIC, Spielberger} 1973) The STAIC is one of the most frequently used selfreport instruments for evaluating children's anxiety. The questionnaire consists of two 20-item subscales: the State Anxiety Scale (S-Anxiety) which measures the current state of anxiety, and the Trait Anxiety Scale (T-Anxiety) which evaluates the generalized, relatively stable tendency to be anxious. In the present study, we used only the T-Anxiety subscale of the STAIC (adapted to Polish by Sosnowski et al. 1989), because our predictions referring to anxiety were based on the tripartite model that addresses the relationship between PA, NA and recurring rather than momentary state of anxiety (Joiner et al. 1999; Watson et al. 1995). Responses to each item were rated on a 3-point scale ranging from hardly ever to often. Higher scores indicated greater anxiety. In the current sample, the Cronbach's $\alpha$ was .86.

\section{Children's Depression Inventory-2nd Edition (CDI-2, Kovacs} 2015) The CDI-2 is a recently revised 28 -item self-report scale designed to assess depressive symptoms in youth aged 7 to 17 years. For each item, a child is instructed to choose the statement (out of the three listed) that most accurately describes his/her thoughts, feelings, or behaviors for the past two weeks. In the current study, the Polish adaptation of a short, 12-item version of the CDI-2 was used (WrocławskaWarchala and Wujcik 2017) that yields a total score comparable to the one produced by the full-length version. Higher scores indicate more severe symptoms of depression. In the current sample, the Cronbach's $\alpha$ was .79.

\section{Procedure}

All data collection procedures were reviewed and approved by the Ethics Committee of the University of Lodz, Poland. The study was conducted in accordance with the approved protocol. Principals and counselors provided permission to conduct research in their schools. Prior to the study, meetings were held with parents at participating schools where they were invited to allow their children to complete a set of questionnaires about their feelings. Parents were provided with access to all measures, if requested, and assured that the data collected would remain confidential. Children for whom parental consent was obtained completed all instruments in their classrooms during school hours. Children for whom consent was not obtained remained in the classroom, but did not participate in the study (they were provided with alternative activities by their teachers). The teachers were also present in the classrooms, but they did not take part in data collection.
The students were supervised by a psychologist (one of the authors) who first carefully instructed them how to complete each questionnaire and ensured that they understood the instructions. Next, the psychologist asked students to give oral assent and administered the questionnaires. Students were informed that they should place all completed questionnaires in the envelopes provided so that neither their teachers nor the supervising psychologist would be aware of their individual responses. Students completed the Polish version of the PANAS-C along with the CDI-2 and the T-Anxiety subscale of the STAIC and sealed them in the envelopes.

\section{Data Analytic Strategy}

Due to its size, the sample was randomly split into a scale examination subsample $\left(n=361 ; M_{\text {age }}=11.88 ; 189\right.$ boys and 172 girls; 109 fourth graders, 122 fifth graders, and 130 sixth graders), and a scale validation subsample $(n=361$; $M_{\text {age }}=12.01 ; 190$ boys and 171 girls; 108 fourth graders, 123 fifth graders, and 130 sixth graders). The samples differed neither in the proportion of boys and girls, $\chi^{2}(1, N=$ $722)=.006, p=.941$, nor in the proportion of fourth, fifth, and sixth graders, $\chi^{2}(2, N=722)=.009, p=.996$. The proportions in the subsamples closely mirrored those in the overall sample. The STAIC and CDI-2 scores also did not differ between the samples. ${ }^{2}$

The scale examination subsample was used to determine whether the psychometric properties of the Polish version were similar to the original, English version of the scale. We employed the analytical approach used in the development of the PANAS-C by Laurent et al. (1999), and subsequently by Stevanovic et al. (2013) and Ciucci et al. (2017) in the development of the Serbian and Italian versions of the PANAS-C, respectively. Specifically, we first calculated corrected itemtotal correlations for all items on the NA and PA scales and performed an exploratory factor analysis (EFA) using SPSS in which the principal axis method was chosen, requesting a 2factor solution. We also inspected the scree plot in order to ensure that this solution fit the data. Following the example of previous studies (Ciucci et al. 2017; Kiernan et al. 2001; Stevanovic et al. 2013), we retained an item if its corrected item-total correlation was .30 and above and its factor loading was .40 and above (see also Gorsuch 1997; Nunnally and Bernstein 1994). We used a varimax rotation because PA and NA have been conceptualized as two independent factors (Watson and Tellegen 1985). Moreover, evidence suggests that they are orthogonal or only weakly correlated (Watson 2000; Yik et al. 2011).

\footnotetext{
${ }^{2}$ After conducting a series of confirmatory factor analyses, we also compared the subsamples with regard to the PANAS-C scales. The analysis showed no significant differences.
} 
To further examine the structure of the Polish PANAS$\mathrm{C}$, we performed a series of confirmatory factor analyses (CFAs) in AMOS with the maximum likelihood estimation method, using the scale validation subsample. To evaluate the fit of the tested models we relied on the most frequently recommended indices: comparative fit index (CFI), the Tucker-Lewis index (TLI), and the root mean square error of approximation (RMSEA) (Jackson et al. 2009). We used traditional cutoff values: CFI and TLI indices in the .90 range and a RMSEA of less than .06 (Hooper et al. 2008; Marsh et al. 1996). To improve model fit, all items with factor loadings below .40 were eliminated (see Ebesutani et al. 2011b, for a similar strategy). We decided not to use any other modifications to improve model fit (e.g., correlating error terms or cross-loadings), based on the recent recommendations concerning such practices (Hermida 2015). To compare competing models, we used chi-square difference tests (Steiger et al. 1985).

As already mentioned, we tested three models suggested in literature (see Fig. 1). Model 1 was proposed by Laurent et al. (1999). It consists of PA and NA factors that emerged in EFA. Model 2 and Model 3 were proposed by Ebesutani and colleagues (Ebesutani et al. 2011a, 2012). The first of these models (Model 2) limits the number of items to five per scale (Ebesutani et al. 2012). The second alternative model (Model 3) is based on the 27 items of the original PANAS-C (Laurent et al. 1999) and consists of two higher-order factors (PA and NA) and two lower-order factors, $\mathrm{NA}_{\text {fear }}$ and NA distress (Ebesutani et al. 2011a). $\mathrm{NA}_{\text {fear }}$ is proposed as a 5-item scale, whereas the remaining 10 NA items compose $\mathrm{NA}_{\text {distress. }}$ The PA scale consists of 12 items. To investigate the reliability of the PA and NA scales, we calculated Cronbach's alpha coefficients, using .70 as the cut-off for acceptable reliability and .80 as the cut-off for good reliability (De Vellis 2003).

Following selection of best-fitting model(s), we calculated the correlation among the PANAS-C scales and traditional measures of anxiety and depression, using the overall sample. We also conducted the hierarchical multiple regression analysis based on the approach used by Laurent et al. (1999). This approach, introduced by Watson et al. (1995), addresses the problem of shared variance between the analyzed constructs by partialling out the variance unrelated to the predicted score. For instance, when we examined the CDI-2 score (criterion measure) predicted by PA, we controlled for non-target measures in the model (i.e., STAIC and NA scores).

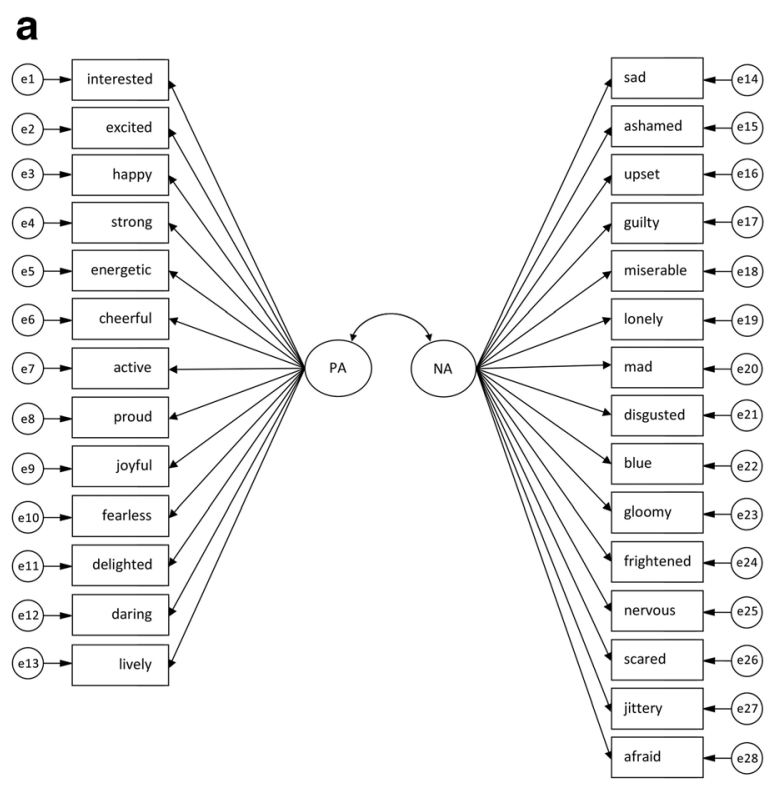

\section{b}

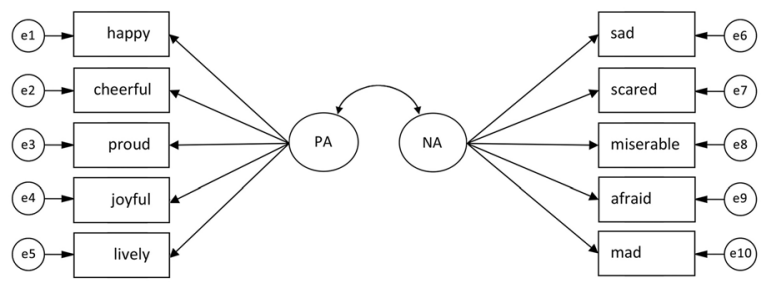

C

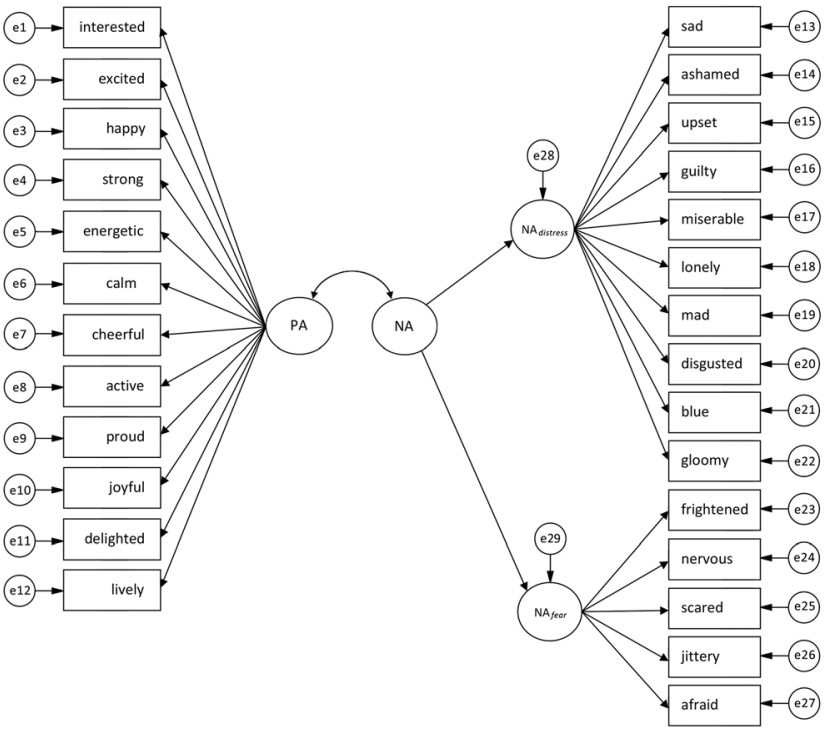

Fig. 1 Models tested in the current study. A. Two-factor original model - longer version. B. Two-factor model - shorter version. C. Second-order model 
Finally, we examined gender and age differences in the PANAS-C scores using 2 (gender) $\times 3$ (school level) MANOVAs with the PANAS-C scores as dependent variables.

\section{Results}

\section{Factor Structure of the PANAS-C}

The EFA performed on the scale examination subsample yielded two easily interpretable factors, PA and NA, which explained $36.54 \%$ of variance. The inspection of the scree plot also supported the two-factor solution. Table 1 presents the corrected item-total correlations and factor loadings for both PA and NA. Two items from the PA scale, alert and calm, did not meet the retention criterion of item-total correlation of $\geq$ .30 and thus were eliminated. This resulted in a 13-item PA scale and a 15-item NA scale. Cronbach's alpha coefficients for these scales were .88 and .90 , respectively. As expected, the scales were weakly negatively correlated, $r(361)=-.33$.

We next performed a series of CFAs, using the scale validation subsample. The first model we tested, that is, the 28item two-factor model that emerged from the EFA (Model 1a), indicated an inadequate fit (see Table 2). Moreover, as revealed in Table 3, two additional items (disgusted and fearless) had factor loadings below .40. Therefore, we eliminated these two items and tested the 26-item model (i.e., a 12item PA scale and a 14-item NA scale; Model 1b). This time the fit was better but RMSEA, CFI and TLI still remained below the standard cut-off criteria we adopted.

These criteria were met by the next model we tested, that is, the 10-item version proposed by Ebesutani et al. (2012). (Model 2; see Table 2). The smaller number of items, however, resulted in slightly lowered internal consistency of the scale, as compared to the 26-item version. Cronbach's alpha coefficients for the PA and NA scales were .89 and .90 for the 26 -item version, and .85 and .74 for the 10 -item version. The correlation between PA and NA was also weaker for the 26item version, $r(361)=-.37$, than for the 10 -item version, $r(361)=-.41$.

The last model examined was based on the 27 items of the original PANAS-C and consisted of two higher-order factors (PA and NA), and two lower-order factors, $\mathrm{NA}_{\text {fear }}$ and $\mathrm{NA}_{\text {distress }}$ (Model 3a). This hierarchical model, proposed by Ebesutani et al. (2011a), slightly differs from the 26 -item version (Model 1b) in the selection of items. Specifically, it does not include daring but includes disgusted and calm (see Fig. 1). We decided to directly replicate Ebesutani and colleagues' model instead of testing the hierarchical structure of the 26item PANAS-C because it is possible that somewhat low CFI and TLI values observed in our previous CFAs resulted from the non-hierarchical structure of the models we tested rather
Table 1 Item-total correlations and factor loadings for the PA and NA scales using the scale examination subsample (exploratory factor analysis)

\begin{tabular}{|c|c|c|c|}
\hline \multirow[t]{2}{*}{ Item } & \multirow{2}{*}{$\begin{array}{l}\text { Item-total } \\
\text { correlation } \\
(n=361)\end{array}$} & \multicolumn{2}{|c|}{$\begin{array}{l}\text { Exploratory factor } \\
\text { analysis }(n=361)\end{array}$} \\
\hline & & PA & NA \\
\hline \multicolumn{4}{|l|}{ NA } \\
\hline Sad (smutny) & .66 & -.21 & .67 \\
\hline Frightened (przestraszony) & .50 & -.01 & .54 \\
\hline Ashamed (zawstydzony) & .46 & -.07 & .48 \\
\hline Upset (zmartwiony) & .62 & -.10 & .64 \\
\hline Nervous (zdenerwowany) & .62 & -.08 & .66 \\
\hline Guilty (winny) & .47 & -.04 & .49 \\
\hline Scared (przerażony) & .61 & -.05 & .64 \\
\hline Miserable (nieszczęśliwy) & .68 & -.21 & .69 \\
\hline Jittery (roztrzęsiony) & .60 & -.05 & .64 \\
\hline Afraid (zaniepokojony) & .60 & .01 & .65 \\
\hline Lonely (samotny) & .53 & -.28 & .53 \\
\hline Mad (wściekły) & .56 & .02 & .61 \\
\hline Disgusted (pełen obrzydzenia) & .42 & .04 & .47 \\
\hline Blue (przygnębiony) & .64 & -.26 & .64 \\
\hline Gloomy (ponury) & .59 & -.17 & .60 \\
\hline \multicolumn{4}{|l|}{ PA } \\
\hline Interested (zainteresowany) & .45 & .46 & -.06 \\
\hline Alert (czujny) & .25 & - & - \\
\hline Excited (podekscytowany) & .39 & .46 & .08 \\
\hline Happy (radosny) & .63 & .62 & -.39 \\
\hline Strong (silny) & .56 & .59 & -.02 \\
\hline Energetic (pełen energii) & .57 & .58 & -.24 \\
\hline Calm (spokojny) & .29 & - & - \\
\hline Cheerful (wesoły) & .63 & .63 & -.32 \\
\hline Active (aktywny) & .50 & .53 & -.08 \\
\hline Proud (dumny) & .60 & .66 & -.01 \\
\hline Joyful (zadowolony) & .57 & .58 & .31 \\
\hline Fearless (nieustraszony) & .49 & .56 & -.11 \\
\hline Delighted (zachwycony) & .62 & .65 & -.09 \\
\hline Daring (odważny) & .59 & .61 & -.18 \\
\hline Lively (pełen życia) & .61 & .62 & -.32 \\
\hline
\end{tabular}

Bold values indicate factor loadings $\geq .40$

than the selection of items. The fit of Model 3a was slightly better than the fit of Model 1b, but RMSEA, CFI and TLI still did not meet the adopted criteria. Moreover, three items (strong, calm and disgusted) had factor loadings below .40. Thus, we excluded these items (Model 3b), which improved the fit, but RMSEA, CFI and TLI reached cut-off values only marginally. The Cronbach's alpha coefficients for the PA, NA, $\mathrm{NA}_{\text {fear }}$ and $\mathrm{NA}_{\text {distress }}$ scales were $.88, .90, .78$, and .86, respectively. As predicted, the correlation between PA and NA was 
Table 2 Examination of five alternative models using the scale validation subsample (goodnessof-fit indexes)

\begin{tabular}{lcccccc}
\hline & $\chi^{2}$ & $d f$ & $\chi^{2} / d f$ & CFI & TLI & RMSEA (90\% CFI) \\
\hline Two-factor models (PA, NA) - longer version & & & & & \\
Model 1a: 28 item-version & 1119.51 & 349 & 3.20 & .812 & .796 & $.078(.073-.083)$ \\
Model 1b: 26-item version & 932.07 & 298 & 3.13 & .836 & .822 & $.077(.071-.082)$ \\
Two-factor model (PA, NA) - shorter version & & & & & \\
Model 2: 10-item version & 78.46 & 34 & 2.31 & .965 & .953 & $.060(.043-.078)$ \\
Second-order models (PA, NA, lower-order & $\mathrm{NA}_{\text {fear }}$ and $\mathrm{NA}_{\text {distress }}$ ) & & & \\
Model 3a: 27-item version & 917.86 & 323 & 2.84 & .845 & .832 & $.072(.066-.077)$ \\
Model 3b: 24-item version & 741.14 & 251 & 2.95 & .864 & .850 & $.074(.068-.080)$ \\
\hline
\end{tabular}

moderate, $r(361)=-.38$, whereas the correlation between $\mathrm{NA}_{\text {fear }}$ and $\mathrm{NA}_{\text {distress }}$ was strong, $r(361)=.69$. Moreover, $\mathrm{NA}_{\text {fear }}$ was weakly related to PA, $r(361)=-.23$, whereas $\mathrm{NA}_{\text {distress }}$ was moderately related to PA, $r(361)=-.42$.

Summing up, the CFA indicated that only one of the tested models, the 10-item two-factor model, indicated an adequate fit, whereas the models based on the larger number of items did not meet the criteria we adopted. Out of these models, Model $1 \mathrm{~b}$ and Model $3 \mathrm{~b}$ fitted the data better than the remaining models. Moreover, fit indices observed for these models were comparable to those reported by Ebesutani et al. (2011a). We thus compared these models using the chi-square difference test. The comparison revealed that Model $3 \mathrm{~b}$ had a significantly better fit than Model 1b $\left(\Delta \chi^{2}(25)=190.93\right.$, $p<0.001$ ). Therefore, we performed all further analyses separately for the 10-item two-factor model (Model 2) and for the 24-item hierarchical model (Model 3b). Descriptive statistics for both versions are given in Table 4 .

\section{The PANAS-C Scales in Relation to Anxiety and Depression}

To provide evidence for convergent and discriminant validity of the PANAS-C scores, we calculated correlations among the PA and NA scales and traditional measures of anxiety and depression, using the overall sample (Table 4). The results demonstrated that PA was negatively related to the symptoms of the two disorders, whereas NA was positively related to these symptoms. The strength of the correlations indicated that these relationships were moderate to high. Moreover, $\mathrm{NA}_{\text {fear }}$ and $\mathrm{NA}_{\text {distress }}$ were related to both anxiety and depression, but the correlations observed for $\mathrm{NA}_{\text {distress }}$ were slightly higher. Importantly, these correlations were similar for the 10and 24-item versions.

To further examine the links between PA, NA and affective disorders, we conducted the hierarchical multiple regression analysis described above. In line with the tripartite model, the results confirmed that NA had a significant partial correlation with both criterion measures (anxiety and depression) when non-target variance was controlled, whereas PA, being unique to depression, had a negative partial correlation with the CDI-
2 score, but a non-significant partial correlation with the STAIC score (see Table 5). Of importance, the pattern of results was similar, regardless of the PANAS-C version.

\section{Age and Gender Differences}

Finally, we examined gender and age differences in the PANAS-C scores. We conducted 2 (gender) $\times 3$ (school level) MANOVAs with the PA and NA scales of the PANAS-C (10item and 24-item versions), $\mathrm{NA}_{\text {fear }}$ and $\mathrm{NA}_{\text {distress }}$ (only 24item-version) as dependent variables (Table 6). As expected, children obtained higher scores on the PA scale than on the NA scale (and its components), regardless of their age or gender. In the case of the NA scale, we observed a significant main effect of gender, $F(1,716)=12.95, p<.001, \eta_{\mathrm{p}}{ }^{2}=.02$ (the 10-item version); $F(1,716)=14.58, p<.001, \eta_{\mathrm{p}}{ }^{2}=.02$ (the 24-item version); and a significant gender by school level interaction, $F(2,716)=5.85, p=.003, \eta_{\mathrm{p}}{ }^{2}=.02($ the 10 -item version); $F(2,716)=5.26, p=.005, \eta_{\mathrm{p}}{ }^{2}=.01$ (the 24-item version). In general, regardless of the version, girls had higher scores on the NA scale than boys, but this effect was stronger for older children than for younger children (i.e., we observed no gender differences among 4th graders but such differences emerged among 5th and 6th graders). This resulted from the fact the levels of NA were lower in older boys than in younger boys, but no age differences were found in girls. The pattern of results was similar for the NA scale components (24-item version). Specifically, we observed a significant main effect of gender for both $\mathrm{NA}_{\text {fear, }} F(1,716)=10.74, p=.001$, $\eta_{\mathrm{p}}{ }^{2}=.02$, and $\mathrm{NA}_{\text {distress }}, F(1,716)=13.80, p<.001$, $\eta_{\mathrm{p}}{ }^{2}=.02$. The interaction between gender and school level was also significant for both $\mathrm{NA}_{\text {fear }}, F(2,716)=3.89$, $p=.021, \eta_{\mathrm{p}}{ }^{2}=.01$, and $\mathrm{NA}_{\text {distress }}, F(2,716)=5.22, p=.006$, $\eta_{\mathrm{p}}{ }^{2}=.01$. In general, the levels of $\mathrm{NA}_{\text {fear }}$ were lower in older boys than in younger boys, but no such effects were found in girls. In the case of $\mathrm{NA}_{\text {distress }}$, the scores were lower in younger girls than older girls but no such effects were observed in boys.

Similar analyses for the PA scale revealed a main effect of school level, $F(2,716)=6.59, p=.001, \eta_{\mathrm{p}}{ }^{2}=.02$ (the 10 -item version); $F(2,716)=6.25, p=.002, \eta_{\mathrm{p}}{ }^{2}=.02$ (the 24-item version), indicating that younger children obtained higher 
Table 3 Factor loadings for the PA and NA scales using the scale validation subsample (confirmatory factor analysis)

\begin{tabular}{|c|c|c|c|c|c|c|c|c|c|c|c|c|}
\hline \multirow[t]{5}{*}{ Item } & \multicolumn{12}{|c|}{ Confirmatory factor analysis $(n=361)$} \\
\hline & \multicolumn{6}{|c|}{ Two-factor model } & \multicolumn{6}{|c|}{ Second-order model } \\
\hline & \multicolumn{4}{|c|}{ Longer version } & \multirow{2}{*}{\multicolumn{2}{|c|}{$\frac{\text { Shorter version }}{10 \text {-item version }}$}} & & & & & & \\
\hline & \multicolumn{2}{|c|}{ 28-item version } & \multicolumn{2}{|c|}{ 26-item version } & & & \multicolumn{3}{|c|}{ 27-item version } & \multicolumn{3}{|c|}{ 24-item version } \\
\hline & PA & NA & $\mathrm{PA}$ & NA & $\mathrm{PA}$ & NA & $\mathrm{PA}$ & $\mathrm{NA}_{\text {fear }}$ & $\mathrm{NA}_{\text {distress }}$ & $\mathrm{PA}$ & $\mathrm{NA}_{\text {fear }}$ & $\mathrm{NA}_{\text {distress }}$ \\
\hline \multicolumn{13}{|l|}{ NA } \\
\hline Sad (smutny) & & .74 & & .75 & & .78 & & & .74 & & & .75 \\
\hline Frightened (przestraszony) & & .51 & & .51 & & - & & .51 & & & .51 & \\
\hline Ashamed (zawstydzony) & & .48 & & .48 & & - & & & .48 & & & .48 \\
\hline Upset (zmartwiony) & & .69 & & .70 & & - & & & .69 & & & .70 \\
\hline Nervous (zdenerwowany) & & .66 & & .66 & & - & & .66 & & & .66 & \\
\hline Guilty (winny) & & .54 & & .54 & & - & & & .54 & & & .54 \\
\hline Scared (przerażony) & & .62 & & .62 & & .57 & & .62 & & & .62 & \\
\hline Miserable (nieszczęśliwy) & & .63 & & .64 & & .69 & & & .63 & & & .64 \\
\hline Jittery (roztrzęsiony) & & .53 & & .53 & & - & & .53 & & & .53 & \\
\hline Afraid (zaniepokojony) & & .59 & & .59 & & .51 & & .59 & & & .59 & \\
\hline Lonely (samotny) & & .67 & & .67 & & - & & & .67 & & & .67 \\
\hline Mad (wściekły) & & .53 & & .53 & & .46 & & & .53 & & & .53 \\
\hline Disgusted (pełen obrzydzenia) & & .36 & & - & & - & & & .36 & & & - \\
\hline Blue (przygnębiony) & & .70 & & .70 & & - & & & .71 & & & .70 \\
\hline Gloomy (ponury) & & .68 & & .68 & & - & & & .68 & & & .68 \\
\hline \multicolumn{13}{|l|}{ PA } \\
\hline Interested (zainteresowany) & .47 & & .47 & & - & & .47 & & & .47 & & \\
\hline Alert (czujny) & - & & - & & - & & - & & & - & & \\
\hline Excited (podekscytowany) & .52 & & .52 & & - & & .51 & & & .52 & & \\
\hline Happy (radosny) & .77 & & .78 & & .81 & & .79 & & & .79 & & \\
\hline Strong (silny) & .43 & & .41 & & - & & .39 & & & - & & \\
\hline Energetic (pełen energii) & .63 & & .63 & & - & & .62 & & & .62 & & \\
\hline Calm (spokojny) & - & & - & & - & & .37 & & & - & & \\
\hline Cheerful (wesoły) & .80 & & .81 & & .85 & & .82 & & & .82 & & \\
\hline Active (aktywny) & .60 & & .60 & & - & & .59 & & & .59 & & \\
\hline Proud (dumny) & .67 & & .67 & & .61 & & .66 & & & .65 & & \\
\hline Joyful (zadowolony) & .71 & & .72 & & .73 & & .72 & & & .73 & & \\
\hline Fearless (nieustraszony) & .33 & & - & & - & & - & & & - & & \\
\hline Delighted (zachwycony) & .66 & & .66 & & - & & .66 & & & .66 & & \\
\hline Daring (odważny) & .52 & & .50 & & - & & - & & & - & & \\
\hline Lively (pełen życia) & .73 & & .74 & & .71 & & .73 & & & .73 & & \\
\hline
\end{tabular}

scores on the PA scale than older children. In the case of the 10 -item version, this effect was qualified by a significant gender by school level interaction, $F(2,716)=3.11, p=.045$, $\eta_{\mathrm{p}}{ }^{2}=.01$. Specifically, the levels of PA were lower in older girls than in younger girls, whereas no age differences were observed in boys. As a result of this effect, girls from the 6th grade scored lower on the PA scale than boys of the same age, whereas in 4th and 5th grades no gender differences were observed.

\section{Discussion}

The goal of the current study was to develop a Polishlanguage version of the PANAS-C and test its psychometric properties in a sample of school children. Additionally, the present study tested two alternative versions of the scale - a 10-item PANAS-C (Ebesutani et al. 2012) and a second-order PANAS-C (Ebesutani et al. 2011a). So far, the first of these alternative versions has been tested only in two countries (the 
Table 4 Descriptive statistics and correlations among the PANAS-C scales and measures of anxiety and depression

\begin{tabular}{|c|c|c|c|c|c|c|}
\hline & 1 & 2 & 3 & 4 & 5 & 6 \\
\hline \multicolumn{7}{|c|}{ Two-factor model, 10 -item version } \\
\hline 1. STAIC & - & & & & & \\
\hline 2. CDI-2 & $.71 * * *$ & - & & & & \\
\hline 3. PA & $-.39 * * *$ & $-.56^{* * *}$ & - & & & \\
\hline 4. NA & $.65 * * *$ & $.61 * * *$ & $-.41 * * *$ & - & & \\
\hline$M$ & 34.62 & 5.51 & 19.44 & 10.37 & - & - \\
\hline$S D$ & 7.63 & 3.87 & 4.31 & 4.14 & - & - \\
\hline \multicolumn{7}{|c|}{ Second-order model, 24-item version } \\
\hline 1. STAIC & - & & & & & \\
\hline 2. CDI-2 & $.71 * * *$ & - & & & & \\
\hline 3. PA & $-.34 * * *$ & $-.51 * * *$ & - & & & \\
\hline 4. NA & $.70 * * *$ & $.66^{* * *}$ & $-.37 * * *$ & - & & \\
\hline 5. $\mathrm{NA}_{\text {fear }}$ & $.60 * * *$ & $.50 * * *$ & $-.24 * * *$ & $.88 * * *$ & - & \\
\hline 6. $\mathrm{NA}_{\text {distress }}$ & $.68 * * *$ & $.68 * * *$ & $-.41 * * *$ & $.96 * * *$ & $.72 * * *$ & - \\
\hline$M$ & 34.62 & 5.51 & 37.58 & 28.04 & 9.81 & 18.23 \\
\hline$S D$ & 7.63 & 3.87 & 7.64 & 10.45 & 4.11 & 7.11 \\
\hline
\end{tabular}

$* * * p<.001$

United States and Spain), whereas the second version has been tested only in the U.S.

In general, the results of EFA closely mirrored those reported for the original PANAS-C (Laurent et al. 1999) and previous translations (e.g., Ciucci et al. 2017; Kiernan et al. 2001; Stevanovic et al. 2013). Specifically, the PANAS-C scales loaded on two separate factors that were easily interpretable as PA and NA, internally consistent, and weakly negatively correlated. The structure of the PANAS-C was further examined with CFA that, except for two studies (Ciucci et al. 2017; Sanmartín et al. 2018), was not used in previous translation studies validating the long version of the PANAS-C. CFA confirmed that affect, as measured with the PANAS-C, consists of two general components, previously identified in

Table 5 Hierarchical multiple regression examining partial correlations of the PA and NA scales with measures of anxiety (STAIC) and depression (CDI2)

\begin{tabular}{|c|c|c|c|c|}
\hline \multirow[t]{2}{*}{ Criterion measure } & \multirow[t]{2}{*}{ Nontarget measure } & \multicolumn{2}{|c|}{ Partials in block 1} & \multirow[t]{2}{*}{ PA partia } \\
\hline & & Nontarget & NA & \\
\hline \multicolumn{5}{|c|}{ Two-factor model, 10 -item version } \\
\hline STAIC-Trait & CDI-2 & $.49^{* * *}$ & $.40 * * *$ & .05 \\
\hline CDI-2 & STAIC & $.49 * * *$ & $.21 * * *$ & $-.40 * * *$ \\
\hline \multicolumn{5}{|c|}{ Second-order model, 24-item version } \\
\hline STAIC-Trait & $\mathrm{CDI}-2$ & $.44 * * *$ & $.45^{* * *}$ & .06 \\
\hline $\mathrm{CDI}-2$ & STAIC & $.44 * * *$ & $.28 * * *$ & $-.36 * * *$ \\
\hline
\end{tabular}

$* * * p<.001$ research in adults (Watson and Tellegen 1985; Zevon and Tellegen 1982), but this conclusion was valid only for the 10 -item version. The remaining versions indicated an inadequate fit according to the cut-off criteria we adopted.

The comparison between our findings and the results of previous studies that employed CFA to test the structure of the "long" PANAS-C (e.g., Bushman and Crowley 2010; Ciucci et al. 2017; Ebesutani et al. 2011a, 2011b) or the original PANAS in youth (Allan et al. 2015; Ortuño-Sierra et al. 2015) sheds some light on the reasons why Model 1 and Model 3 demonstrated an inadequate fit in our study. First, previous studies improved the fit of the tested models by allowing correlated errors between some items (Allan et al. 2015; Ortuño-Sierra et al. 2015). Notably, research on factorial structure of the original PANAS in adults also demonstrated that solutions with correlated errors fit data best (e.g., Crawford and Henry 2014; Merz et al. 2013; Terracciano et al. 2003). Following recommendations by Hermida (2015), we decided not to use any modifications that were not theoretically prespecified, although modification indices suggested that allowing measurement errors to correlate may improve model fit. Second, studies that did not allow error terms to correlate, adopted less conservative cut-off criteria than those adopted in our study. For instance, RMSEA and CFI values for best-fitting models ranged from .07 . to .15 (Bushman and Crowley 2010; Ciucci et al. 2017; Ebesutani et al. 2011b) and .77 to .85 , respectively (Ebesutani et al. 2011b). Third, some researchers limited their CFAs to children older than 11 years (e.g., Ebesutani et al. 2011a) or conducted CFAs on the PANAS-C combined with the Physiological Hyperarousal Scale for Children (PH-C) (Ciucci et al. 2017). Taken together, these results demonstrate that when (1) error terms are not allowed to correlate, (2) at least part of the sample consists of children younger than 11, and (3) the PANAS-C is not combined with the PH-C, the "long" version of the scale may not meet traditional conservative cutoff criteria for fit indices.

We should also note that our study was the first to investigate the hierarchical structure of the PANAS-C outside the U.S. The examination of the second-order 27-item model proposed by Ebesutani et al. (2011a) indicated that three items (strong, calm, and disgusted) had low factor loadings, and thus we removed them. This resulted in a 24-item hierarchical version of the PANAS-C that consists of two broad dimensions of affect (PA and NA) and two highly correlated NA subdimensions $\left(\mathrm{NA}_{\text {fear }}\right.$ and $\left.\mathrm{NA}_{\text {distress }}\right)$. Although, as already mentioned, the fit of this 24-item model was better than the fit of other full-length models tested in our study, it still did not meet the adopted criteria. Moreover, it was slightly worse than the fit of the hierarchical model reported by Ebesutani et al. (2011a). There might be at least two reasons for these discrepancies. First, Ebesutani et al. conducted CFA on the 15 NA items only, whereas we tested the structure of both the PA and 
Table 6 Means and standard deviations by gender and school level for the PANAS-C scales

\begin{tabular}{|c|c|c|c|c|c|c|}
\hline & \multicolumn{3}{|l|}{ Girls } & \multicolumn{3}{|l|}{ Boys } \\
\hline & $\begin{array}{l}\text { 4th graders } \\
(n=91)\end{array}$ & $\begin{array}{l}5 \text { th graders } \\
(n=104)\end{array}$ & $\begin{array}{l}\text { 6th graders } \\
(n=148)\end{array}$ & $\begin{array}{l}\text { 4th graders } \\
(n=126)\end{array}$ & $\begin{array}{l}5 \text { th graders } \\
(n=141)\end{array}$ & $\begin{array}{l}\text { 6th graders } \\
(n=112)\end{array}$ \\
\hline \multicolumn{7}{|c|}{ Two-factor model, 10 -item version } \\
\hline $\mathrm{PA}$ & $20.56(3.62)$ & $19.27(4.10)$ & $18.18(4.92)$ & $20.10(3.62)$ & $19.44(4.24)$ & $19.63(4.61)$ \\
\hline NA & $10.53(4.21)$ & $10.78(4.12)$ & $11.41(4.59)$ & $10.70(4.25)$ & $9.70(3.34)$ & $8.99(3.83)$ \\
\hline \multicolumn{7}{|c|}{ Second-order model, 24-item version } \\
\hline PA & $39.43(7.29)$ & $37.42(7.30)$ & $35.51(8.52)$ & $38.65(6.35)$ & $37.69(7.41)$ & $37.61(8.11)$ \\
\hline NA & $28.10(10.44)$ & $29.31(10.52)$ & $30.97(11.86)$ & $28.23(10.20)$ & $26.34(8.32)$ & $24.90(10.05)$ \\
\hline $\mathrm{NA}_{\text {fear }}$ & $10.07(4.33)$ & $10.48(4.52)$ & $10.40(4.41)$ & $10.25(4.31)$ & $9.17(3.15)$ & $8.52(3.58)$ \\
\hline $\mathrm{NA}_{\text {distress }}$ & $18.03(6.74)$ & $18.83(6.70)$ & $20.57(8.39)$ & $17.98(6.71)$ & $17.17(6.00)$ & $16.38(6.94)$ \\
\hline
\end{tabular}

Standard deviations are given in the parentheses

NA scales. Second, Ebesutani et al. performed CFA only in the sample of children aged 12 to 17 (excluding children aged 6 to 11), while our sample consisted of younger students. Hence, the notion that NA, as measured with the PANAS-C, similarly to NA measured with the adult PANAS (Gaudreau et al. 2006; Killgore 2000), might be divided into lower-order components warrants further investigation.

Overall, our results suggest that the underlying structure of the PANAS-C, similar to the structure of the original PANAS (e.g., Allan et al. 2015; Crawford and Henry 2014; Leue and Beauducel 2011), may be more complex than initially proposed. This might be related to the fact that the PANAS-C (similar to the original PANAS) contains some correlated, redundant items, which may explain why the shorter version of the scale fitted the data better than the "long" PANAS-C not only in our study but also in previous studies (Ebesutani et al. 2012; Sanmartín et al. 2018; see also Damásio et al. 2013 for a CFA of an 8-item version of the PANAS-C). This observation is in line with a general tendency to shorten the PANAS-C by removing poorly performing items. Overall, all translations of the PANAS-C are shorter than their original 27-item counterpart (from the 24-item Japanese and Italian versions [Yamasaki et al. 2006; Ciucci et al. 2017, respectively] to the 21-item Serbian version [Stevanovic et al. 2013]; see also Damásio et al. 2013). Our analyses also suggested that some of the PANAS-C items should be eliminated. Specifically, the EFA revealed that two items, alert and calm, are problematic due to low item-total correlations, whereas CFAs identified additional items, disgusted, fearless, and strong, with low factor loadings. A comparison across translations of the PANAS$\mathrm{C}$ indicated that these results closely correspond to those of previous validation studies (Ciucci et al. 2017; Laurent et al. 1999; Stevanovic et al. 2013; Yamasaki et al. 2006). Alert and fearless were eliminated from the original PANAS-C as well as from the Italian, Serbian, and Japanese translations. Calm was removed from the Italian translation, whereas disgusted was removed from the Italian and Serbian translations. Moreover, three of the eliminated items (alert, calm, and fearless) were also excluded by Kiernan et al. (2001) in their cross-cultural study conducted with children from 16 European countries. The only exception was strong that was removed only from the Polish version of the PANAS-C. Yet, the factor loading for this adjective was inadequate only when the hierarchical model was tested.

It is possible that whether a particular item was included in or excluded from the scale depended on the frequency of usage of this word in a given culture. For instance, the National Corpus of Polish (i.e., a large collection of books, scientific journal articles, newspapers and other texts in Polish; http://www.nkjp.pl/) indicates that three items removed from the Polish version (disgusted, fearless, alert) are less frequently used ( $<5000$ records) in contemporary Polish than the remaining items (from 4000 to 49,000 records). The exceptions were calm (30,996 records) and strong (34,011 records), but a closer analysis of the meaning of these words suggests that they are used less frequently in emotional contexts than in other contexts. Specifically, in Polish calm is used not only to describe feelings but also to tell others (especially children) to behave appropriately (i.e., in a phrase that can be translated to "Behave yourself"). Thus, children may associate this word with well-behaved rather than peaceful and quiet. In a similar vein, strong is typically used to refer to physical (rather than psychological) strength. Given that children develop vocabulary based on the way the words are used in the culture they grow up, they also describe emotions in a way specific to this culture (Wierzbicka 1999). This may explain why the items that are used occasionally or frequently appear in unemotional contexts were excluded from the PANAS-C.

Taken together, these results suggest that there is some cultural variation in the content of the PA and NA scales, which might be related to general differences in emotional 
experiences across cultures (Eid and Diener 2001). Moreover, the fact that fit indices were better for the shorter than for longer PANAS-C suggests that the former might perform better in child populations. This might be particularly important in groups of younger children who may lack the ability to differentiate between various items that overlap in meaning and to precisely describe their affective experience (Bushman and Crowley 2010). Notably, PA and NA are more highly correlated in younger than in older children and become more distinct with age (Lonigan et al. 1999; Ollendick et al. 2003). Moreover, the structure of the PANAS-C or the original PANAS was found to be particularly difficult to capture in children younger than 11 (Allan et al. 2015; Ebesutani et al. 2011a; Lonigan et al. 1999). It seems also likely that long scales can be difficult or boring to children whose ability to concentrate on the task at hand might be limited. Alternatively, the PANAS-C is often included with other measures in studies, so brevity may be a desirable characteristic of child measures, in general. At the same time, it should be noted that shorter scales typically have large measurement error (Emons et al. 2007), and thus should be treated with caution. For instance, in our study, the 5-item NA scale demonstrated lower internal consistency than the "long" NA scale. However, the remaining psychometric characteristics did not differ between both versions (i.e., the 10-item PANAS and the 24-item PANAS-C), despite differences in factor structures and fit indices. Specifically, regardless of the version, the Polish-language PANAS-C scales were related to traditional measures of anxiety and depression. The observed pattern of results was entirely consistent with the assumptions of the tripartite model which holds that an absence or substantial reduction of PA is unique to depression, whereas general NA is a component shared by both disorders (Clark and Watson 1991; Watson 2000). In line with this assumption and previous studies on the role of PA and NA in anxiety and depression, we demonstrated that NA had significant partial correlations with both disorders, whereas PA had a negative partial correlation only with the measure of depression.

The analysis of gender differences in the PANAS-C scores also confirmed that, regardless of the length, the Polishlanguage version was similar to other language translations (Ciucci et al. 2017; Stevanovic et al. 2013). Specifically, we observed no gender differences in the PANAS-C scores in younger students, but such differences emerged in older students (i.e., older girls obtained higher scores on the NA scale and lower on the PA scale than older boys). These differences resulted from the fact that the levels of NA were lower for older boys than for younger boys and the levels of PA were lower for older girls than for younger girls. Moreover, the analysis of gender differences in the components of NA revealed that older boys reported lower levels of $\mathrm{NA}_{\text {fear }}$ than younger boys, whereas older girls reported higher levels of $\mathrm{NA}_{\text {distress }}$ than younger girls. In general, these findings support the notion that gender differences in depression that is characterized by high levels of NA and low levels of PA, emerge in adolescence (Hankin et al. 2008). Notably, gender differences in NA emerged in younger children (5th grade) than gender differences in PA (6th grade).

Although the present research contributes to a growing literature on the translation of the PANAS-C, it is not without limitations. We recruited our participants from schools located in one of the biggest Polish cities, so smaller, rural communities are not represented. Moreover, the sample did not include older adolescents. Future research would thus benefit from more representative sampling of students. This seems particularly important with respect to age, because, as already mentioned, changes in affect and its structure may emerge in adolescence (Ebesutani et al. 2011b, 2012). Another limitation of the current study is the absence of students from clinical settings. The inclusion of such participants in future studies would allow the utility of the Polish PANAS-C in clinical groups to be tested. Further, although our predictions regarding the relations of the PA and NA scales to traditional measures of anxiety and depression were based on the tripartite model, we did not include any measure of physiological hyperarousal (PH). This enabled us to minimize the time students spent on completing the questionnaires, but limited our conclusions to two components of the tripartite model. Thus, future studies may be supplemented with measures of PH. Given that such studies may rely on the shorter version of the PANAS-C, this should not significantly lengthen the time needed to complete all measures.

Overall, the Polish PANAS-C appears to be a promising complement to the English-language versions and other language translations of the scale. Therefore, it can be used in the same ways as the original measure. This is an important contribution to promoting cross-national studies on affect in children. Of importance, our study expands on previous validation studies by going beyond exploratory factor analyses and testing the properties of two alternative versions of the PANAS-C, the 10-item two-dimensional version and the 24 -item hierarchical version. Although our analyses show that the underlying structure of affect was easier to capture when the shorter version was used, the two versions performed similarly when it comes to the remaining psychometric characteristics. Moreover, the comparison between our results and the results of other studies shows that poor fit indices might be a problem that goes beyond the Polish version and encompasses the "long" PANAS-C and the original PANAS in general. Thus, although based on the CFA results the short version of the scale should be preferred over the longer one, especially when time is limited (e.g., a battery is long, which increases the chance of a child getting bored), the longer version may be used with caution when time and battery length are of no great concern 
and additional information about two components of NA is needed. This new direction in studies on the structure of affect opens new possibilities in exploring the role of fear and distress in the development of emotional disorders.

Acknowledgements The data that support the findings of this study are openly available in Open Science Framework at https://osf.io/vq9ng.

Funding This research was supported by a grant from the Faculty of Educational Sciences, University of Lodz awarded to Monika Wróbel, Maria Finogenow, and Paulina Szymańska.

\section{Compliance with Ethical Standards}

Conflict of Interest Monika Wróbel, Maria Finogenow, Paulina Szymańska, and Jeff Laurent declare that they have no conflict of interest.

Experiment Participants All procedures performed in the current study involving human participants were in accordance with the ethical standards of the institutional and/or national research committee, with the 1964 Helsinki Declaration and its later amendments or comparable ethical standards, and with the ethical principles of the American Psychological Association.

Informed consent Informed consent was obtained from the parents/ guardians of the students who participated in the study, and oral assent was obtained from each student.

Open Access This article is distributed under the terms of the Creative Commons Attribution 4.0 International License (http:// creativecommons.org/licenses/by/4.0/), which permits unrestricted use, distribution, and reproduction in any medium, provided you give appropriate credit to the original author(s) and the source, provide a link to the Creative Commons license, and indicate if changes were made.

Publisher's Note Springer Nature remains neutral with regard to jurisdictional claims in published maps and institutional affiliations.

\section{References}

Allan, N. P., Lonigan, C. J., \& Phillips, B. M. (2015). Examining the factor structure and structural invariance of the PANAS across children, adolescents, and young adults. Journal of Personality Assessment, 97, 616-625. https://doi.org/10.1080/00223891.2015. 1038388.

Beaton, D. E., Bombardier, C., Guillemin, F., \& Ferraz, M. B. (2000). Guidelines for the process of cross-cultural adaptation of self-report measures. Spine, 25, 3186-3191. https://doi.org/10.1097/ 00007632-200012150-00014.

Behr, D. (2017). Assessing the use of back translation: The shortcomings of back translation as a quality testing method. International Journal of Social Research Methodology, 20, 573-584. https://doi.org/10. 1080/13645579.2016.1252188.

Bushman, B. B., \& Crowley, S. L. (2010). Is the structure of affect similar for younger and older children? Cross-sectional differences in negative and positive affectivity. Journal of Psychoeducational Assessment, 28, 31-39. https://doi.org/10.1177/ 0734282909337584 .
Casuso, L., Gargurevich, R., Van den Noortgate, W., \& Van den Bergh, O. (2016). Psychometric properties of the positive and negative affect scale for children (PANAS-C) in Peru. Interamerican Journal of Psychology, 50, 170-185.

Chorpita, B. F., \& Daleiden, E. L. (2002). Tripartite dimensions of emotion in a child clinical sample: Measurement strategies and implications for clinical utility. Journal of Consulting and Clinical Psychology, 70, 1150-1160. https://doi.org/10.1037/0022-006X. 70.5.1150.

Ciucci, E., Baroncelli, A., Tambasco, G., Laurent, J., Catanzaro, S. J., \& Joiner, T. E., Jr. (2017). Measuring positive affect, negative affect, and physiological hyperarousal among Italian youth: Translations of the PANAS-C and PH-C. Journal of Psychopathology and Behavioral Assessment, 39, 373-382. https://doi.org/10.1007/ s10862-017-9596-8.

Clark, L. A., \& Watson, D. (1991). Tripartite model of anxiety and depression: Psychometric evidence and taxonomic implications. Journal of Abnormal Psychology, 100, 316-336. https://doi.org/ 10.1037/0021-843X.100.3.316.

Crawford, J. R., \& Henry, J. D. (2014). The positive and negative affect schedule (PANAS): Construct validity, measurement properties and normative data in a large non-clinical sample. British Journal of Clinical Psychology, 43, 245-265. https://doi.org/10.1348/ 0144665031752934.

Dagys, N., McGlinchey, E. L., Talbot, L. S., Kaplan, K. A., Dahl, R. E., \& Harvey, A. G. (2012). Double trouble? The effects of sleep deprivation and chronotype on adolescent affect. Journal of Child Psychology and Psychiatry, 53, 660-667. https://doi.org/10.1111/j. 1469-7610.2011.02502.x.

Damásio, B. F., Pacico, J. C., Poletto, M., \& Koller, S. H. (2013). Refinement and psychometric properties of the eight-item Brazilian Positive and Negative Affective Schedule for Children (PANAS-C8). Journal of Happiness Studies, 14, 1363-1378. https://doi.org/10.1007/s10902-012-9383-x.

De Vellis, R. F. (2003). Scale development: Theory and applications (2nd ed., Vol. 26). Thousand Oaks: Sage Publications.

Diener, E. (1999). Introduction to the special section on the structure of emotion. Journal of Personality and Social Psychology, 76, 803804. https://doi.org/10.1037/0022-3514.76.5.803.

Ebesutani, C., Smith, A., Bernstein, A., Chorpita, B. F., Higa-McMillan, C., \& Nakamura, B. (2011a). A bifactor model of negative affectivity: Fear and distress components among younger and older youth. Psychological Assessment, 23, 679-691. https://doi.org/10.1037/ a0023234.

Ebesutani, C., Okamura, K., Higa-McMillan, C., \& Chorpita, B. F. (2011b). A psychometric analysis of the positive and negative affect schedule for children-parent version in a school sample. Psychological Assessment, 23, 406-416. https://doi.org/10.1037/ a0022057.

Ebesutani, C., Regan, J., Smith, A., Reise, Higa-McMillan, C., \& Chorpita, B. F. (2012). The 10-item positive and negative affect schedule for children, child and parent shortened versions: Application of item response theory for more efficient assessment. Journal of Psychopathology and Behavioral Assessment, 34, 191203. https://doi.org/10.1007/s10862-011-9273-2.

Eid, M., \& Diener, E. (2001). Norms for experiencing emotions in different cultures: Inter- and intranational differences. Journal of Personality and Social Psychology, 81, 869-885. https://doi.org/ 10.1037//0022-3514.81.5.869.

Emons, W. H. M., Sijtsma, K., \& Meijer, R. R. (2007). On the consistency of individual classification using short scales. Psychological Methods, 12, 105-120. https://doi.org/10.1037/1082-989X.12.1. 105.

Felver, J. C., Butzer, B., Olson, K. J., Smith, I. M., \& Khalsa, S. B. B. (2015). Yoga in public school improves adolescent mood and affect. 
Contemporary School Psychology, 19, 184-192. https://doi.org/10. 1007/s40688-014-0031-9.

Gaudreau, P., Sanchez, X., \& Blondin, J. P. (2006). Positive and negative affective states in a performance-related setting. European Journal of Psychological Assessment, 22, 240-249. https://doi.org/10.1027/ 1015-5759.22.4.240.

Gorsuch, R. L. (1997). Exploratory factor analysis: Its role in item analysis. Journal of Personality Assessment, 68, 532-560. https://doi. org/10.1207/s15327752jpa6803_5.

Gudmundsson, E. (2009). Guidelines for translating and adapting psychological instruments. Nordic Psychology, 61, 29-45. https://doi. org/10.1027/1901-2276.61.2.29.

Hankin, B. L., Wetter, E., \& Cheely, C. (2008). Sex differences in child and adolescent depression: A developmental psychopathological approach. In J. R. Z. Abela \& B. L. Hankin (Eds.), Handbook of depression in children and adolescents (pp. 377-414). New York: Guilford.

Hermida, R. (2015). The problem of allowing correlated errors in structural equation modeling: Concerns and considerations. Computational Methods in Social Sciences, 3, 5-17.

Hooper, D., Coughlan, J., \& Mullen, M. R. (2008). Structural equation modeling: Guidelines for determining model fit. The Electronic Journal of Business Research, Methods, 6, 53-60.

Hughes, A. A., \& Kendall, P. C. (2009). Psychometric properties of the positive and negative affect scale for children (PANAS-C) in children with anxiety disorders. Child Psychiatry and Human Development, 40, 343-352. https://doi.org/10.1007/s10578-0090130-4.

Jackson, D. L., Gillaspy, J. A., Jr., \& Purc-Stephenson, R. (2009). Reporting practices in confirmatory factor analysis: An overview and some recommendations. Psychological Methods, 14, 6-23. https://doi.org/10.1037/a0014694.

Jacques, H. A. K., \& Mash, E. (2004). A test of the tripartite model of anxiety and depression in elementary and high school boys and girls. Journal of Abnormal Child Psychology, 32, 13-25. https://doi.org/ 10.1023/B:JACP.0000007577.38802.18.

Joiner, T. E., Jr., Catanzaro, S. J., \& Laurent, J. (1996). A confirmatory factor-analytic investigation of the tripartite model of depression and anxiety in college students. Cognitive Therapy and Research, 105, 401-409. https://doi.org/10.1007/BF02227911.

Joiner, T. E., Jr., Steer, R. A., Beck, A. T., Schmidt, N. B., Rudd, M. D., \& Catanzaro, S. J. (1999). Physiological hyperarousal: Construct validity of a central aspect of the tripartite model of depression and anxiety. Journal of Abnormal Psychology, 108, 290-298. https:// doi.org/10.1037/0021-843X.108.2.290.

Kiernan, G., Laurent, J., Joiner, T. E. J., Catanzaro, S. J., \& MacLachlan, M. (2001). Cross-cultural examination of the tripartite model with children: Data from the Barretstown studies. Journal of Personality Assessment, 77, 359-379. https://doi.org/10.1207/ S15327752JPA7702 15.

Killgore, W. D. S. (2000). Evidence for a third factor on the positive and negative affect schedule in a college student sample. Perceptual and Motor Skills, 90, 147-152. https://doi.org/10.2466/pms.2000.90.1. 147.

Kovacs, M. (2015). Children's depression inventory (CDI and CDI 2). The Encyclopedia of Clinical Psychology. https://doi.org/10.1002/ 9781118625392.wbecp419.

Laurent, J., Catanzaro, S.J., Joiner, T. E. J., Rudolph, K. D., Potter, K. I., Lambert, S., ... Gathright, T. (1999). A measure of positive and negative affect for children: Scale development and preliminary validation. Psychological Assessment, 11, 326-338. https://doi.org/10. 1037/1040-3590.11.3.326.

Leue, A., \& Beauducel, A. (2011). The PANAS structure revisited: On the validity of a bifactor model in community and forensic samples. Psychological Assessment, 23, 215-225. https://doi.org/10.1037/ a0021400.
Lonigan, C. J., Hooe, E. S., David, C. F., \& Kistner, J. A. (1999). Positive and negative affectivity in children: Confirmatory factor analysis of a two-factor model and its relation to symptoms of anxiety and depression. Journal of Consulting and Clinical Psychology, 67, 374-386. https://doi.org/10.1037/0022-006X.67.3.374.

Marsh, H. W., Balla, J. R., \& Hau, K. T. (1996). An evaluation of incremental fit indexes: A clarification of mathematical and empirical properties. In G. A. Marcoulides \& R. E. Schumacker (Eds.), Advanced structural equation modeling techniques (pp. 315-353). Mahwah: Lawrence Erlbaum.

Mauss, I. B., \& Robinson, M. D. (2009). Measures of emotion: A review. Cognition \& Emotion, 23, 209-237. https://doi.org/10.1080/ 02699930802204677.

Merz, E. L., Malcarne, V. L., Roesch, S. C., Ko, C. M., Emerson, M., Roma, V. G., \& Sadler, G. R. (2013). Psychometric properties of positive and negative affect schedule (PANAS) original and short forms in an African American community sample. Journal of Affective Disorders, 151, 942-949. https://doi.org/10.1016/j.jad. 2013.08.011.

Nunnally, J. C., \& Bernstein, I. H. (1994). Psychometric theory (3rd ed.). New York: McGraw-Hill.

Ollendick, T. H., Seligman, L. D., Goza, A. B., Byrd, D. A., \& Singh, K. (2003). Anxiety and depression in children and adolescents: A factor-analytic examination of the tripartite model. Journal of Child and Family Studies, 12, 157-170. https://doi.org/10.1023/A: 1022806731527.

Ortuño-Sierra, J., Santarén-Rosell, M., Albéniz, A. P., \& FonsecaPedrero, E. (2015). Dimensional structure of the Spanish version of the positive and negative affect schedule (PANAS) in adolescents and young adults. Psychological Assessment, 27, 1-9. https://doi. org/10.1037/pas0000107.

Roxas, A. S., \& Glenwick, D. S. (2014). The relationship of psychological mindedness and general coping to psychological adjustment and distress in high-school adolescents. Individual Differences Research, 12, 38-49.

Rush, J., \& Hofer, S. M. (2014). Differences in within- and betweenperson factor structure of positive and negative affect: Analysis of two intensive measurement studies using multilevel SEM. Psychological Assessment, 26, 462-473. https://doi.org/10.1037/ a0035666.

Salk, R. H., Hyde, J. S., \& Abramson, L. Y. (2017). Gender differences in depression in representative national samples: Meta-analyses of diagnoses and symptoms. Psychological Bulletin, 143, 783-822. https://doi.org/10.1037/bul0000102.

Sanmartín, R., Vicent, M., Gonzálvez, C., Inglés, C. J., Díaz-Herrero, Á., Granados, L., \& García-Fernández, J. M. (2018). Positive and negative affect schedule-short form: Factorial invariance and optimistic and pessimistic affective profiles in Spanish children. Frontiers in Psychology, 9, 392. https://doi.org/10.3389/fpsyg.2018.00392.

Sinnamon, G. C. B., Caltabiano, M., \& Baune, B. T. (2013). Differentiating disordered affect in children and adolescents with type 1 diabetes. Journal of Affective Disorders, 147, 51-58. https://doi.org/10.1016/j.jad.2012.10.006.

Smith, G. T., McCarthy, D. M., \& Anderson, K. G. (2000). On the sins of short-form development. Psychological Assessment, 12, 102-111. https://doi.org/10.1037//1040-3590.12.1.102.

Sosnowski, T., Iwaniszczuk, D., \& Spielberger, C. D. (1989). Polska adaptacja Inwentarza Stanu i Cechy Lęku dla Dzieci (STAIC) [polish adaptaion of the state-trait anxiety inventory for children (STAIC)]. Studia Psychologiczne, 27, 67-79.

Sousa, V. D., \& Rojjanasritat, W. (2011). Translation, adaptation and validation of instruments or scales for use in cross-cultural health care research: A clear and user-friendly guideline. Journal of Evaluation in Clinical Practice, 17, 268-274. https://doi.org/10. 1111/j.1365-2753.2010.01434.x. 
Spielberger, C. D. (1973). Preliminary manual for the State-Trait Anxiety Inventory for Children ("How I feel questionnaire"). Palo Alto: Consulting Psychologists Press.

Steiger, J. H., Shapiro, A., \& Browne, M. W. (1985). On the multivariate asymptotic distribution of sequential chi-square statistics. Psychometrika, 50, 253-263. https://doi.org/10.1007/BF02294104.

Stevanovic, D., Laurent, J., \& Lakic, A. (2013). Measuring positive and negative affect and physiological hyperarousal among Serbian youth. Journal of Personality Assessment, 95, 107-117. https:// doi.org/10.1080/00223891.2012.718301.

Terracciano, A., McCrae, R. R., \& Costa, P. T. (2003). Factorial and construct validity of the Italian positive and negative affect schedule (PANAS). European Journal of Psychological Assessment, 19, 131-141. https://doi.org/10.1027//1015-5759.19.2.131.

Ting-Ting, P., Xue-chen, D., Biao, S., Ying, L., Shi-yun, X., \& Xing-yi, F. (2015). Reliability and validity of the Chinese version of the positive and negative affect scale for children. Chinese Journal of Clinical Psychology, 23, 397-400.

Tsao, J. C., Jacob, E., Seidman, L. C., Lewis, M. A., \& Zeltzer, L. K. (2014). Psychological aspects and hospitalization for pain crises in youth with sickle-cell disease. Journal of Health Psychology, 19, 407-416. https://doi.org/10.1177/1359105312471570.

Watson, D. (2000). Mood and temperament. New York: Guilford Press.

Watson, D., \& Clark, L. A. (1984). Negative affectivity: The disposition to experience aversive emotional states. Psychological Bulletin, 96, 465-490. https://doi.org/10.1037/0033-2909.96.3.465.

Watson, D., \& Clark, L. A. (1994). The PANAS-X: Manual for the Positive and Negative Affect Schedule - Expanded Form. Retrieved from http://ir.uiowa.edu/cgi/viewcontent.cgi?article= 1011\&context=psychology_pubs. Accessed 23 Feb 2018.

Watson, D., \& Clark, L. A. (1995). Depression and the melancholic temperament. European Journal of Personality, 9, 351-366. https://doi.org/10.1002/per.2410090505

Watson, D., \& Clark, L. A. (1997). Measurement and mismeasurement of mood: Recurrent and emergent issues. Journal of Personality
Assessment, 68, 267-296. https://doi.org/10.1207/ s15327752jpa6802_4.

Watson, D., \& Tellegen, A. (1985). Toward a consensual structure of mood. Psychological Bulletin, 98, 219-235. https://doi.org/10. 1037/0033-2909.98.2.219.

Watson, D., Clark, L. A., \& Tellegen, A. (1988). Development and validation of brief measures of positive and negative affect: The PANAS scales. Journal of Personality and Social Psychology, 54, 1063-1070. https://doi.org/10.1037/0022-3514.54.6.1063.

Watson, D., Clark, L. A., Weber, K., Assenheimer, J. S., Strauss, M. E., \& McCormick, R. A. (1995). Testing a tripartite model: II. Exploring the symptom structure of anxiety and depression in student, adult, and patient samples. Journal of Abnormal Psychology, 104, 15-25. https://doi.org/10.1037/0021-843X.104.1.15.

Wierzbicka, A. (1999). Emotions across languages and cultures: Diversity and universals. Cambridge: Cambridge University Press.

Wrocławska-Warchala, E., \& Wujcik R. (2017). Zestaw Kwestionariuszy do Diagnozy Depresji u Dzieci i Młodzieży - CDI-2. Podręcznik [Inventories for the assesment of depression in children and adolescents - CDI-2. Manual]. Warszawa: Pracownia Testów Psychologicznych PTP.

Yamasaki, K., Katsuma, R., \& Sakai, A. (2006). Development of a Japanese version of the positive and negative affect schedule for children. Psychological Reports, 99, 535-546. https://doi.org/10. 2466/pr0.99.2.535-546.

Yik, M., Russell, J. A., \& Steiger, J. H. (2011). A 12-point circumplex structure of core affect. Emotion, 11, 705-731. https://doi.org/10. 1037/a0023980.

Zevon, M. A., \& Tellegen, A. (1982). The structure of mood change: An idiographic/nomothetic analysis. Journal of Personality and Social Psychology, 43, 111-122. https://doi. org/10.1037/0022-3514.43.1.111. 